\title{
Making the diagnosis of Sjögren's syndrome in patients with dry eye
}

\author{
This article was published in the following Dove Press journal: \\ Clinical Ophthalmology \\ 24 December 2015 \\ Number of times this article has been viewed
}

\author{
Kenneth A Beckman ${ }^{1,2}$ \\ Jodi Luchs ${ }^{3,4}$ \\ Mark S Milner 5,6 \\ 'Comprehensive EyeCare of Central \\ Ohio, Westerville, $\mathrm{OH},{ }^{2}$ The Ohio \\ State University, Columbus, $\mathrm{OH}$, \\ ${ }^{3}$ Hofstra University School of \\ Medicine, Hempstead, NY, ${ }^{4}$ South \\ Shore Eye Care, Wantagh, NY, ${ }^{5}$ The \\ Eye Center of Southern Connecticut, \\ PC, Hamden, CT, ${ }^{6}$ Yale University \\ School of Medicine, New Haven, \\ CT, USA
}

\begin{abstract}
Sjögren's syndrome (SS) is a chronic and progressive systemic autoimmune disease that often presents initially with symptoms of dry eye and dry mouth. Symptoms are often nonspecific and develop gradually, making diagnosis difficult. Patients with dry eye complaints warrant a step-wise evaluation for possible SS. Initial evaluation requires establishment of a dry eye diagnosis using a combination of patient questionnaires and objective ocular tests, including inflammatory biomarker testing. Additional work-up using the Schirmer test and tear film break-up time can differentiate between aqueous-deficient dry eye (ADDE) and evaporative dry eye. The presence of ADDE should trigger further work-up to differentiate between SS-ADDE and non-SS-ADDE. There are numerous non-ocular manifestations of SS, and monitoring for SS-related comorbid findings can aid in diagnosis, ideally in collaboration with a rheumatologist. The clinical work-up of SS can involve a variety of tests, including tear function tests, serological tests for autoantibody biomarkers, minor salivary gland and lacrimal gland biopsies. Examination of classic SS biomarkers (SS-A/Ro, SS-B/La, antinuclear antibody, and rheumatoid factor) is a convenient and non-invasive way of evaluating patients for the presence of SS, even years prior to confirmed diagnosis, although not all SS patients will test positive, particularly those with early disease. Recently, newer biomarkers have been identified, including autoantibodies to salivary gland protein-1, parotid secretory protein, and carbonic anhydrase VI, and may allow for earlier diagnosis of SS. A diagnostic test kit is commercially available $\left(\mathrm{Sjö}{ }^{\circledR}\right)$, incorporating these new biomarkers along with the classic autoantibodies. This advanced test has been shown to identify SS patients who previously tested negative against traditional biomarkers only. All patients with clinically significant ADDE should be considered for serological assessment for SS, given the availability of new serological diagnostic tests and the potentially serious consequences of missing the diagnosis.
\end{abstract}

Keywords: dry eye, Sjögren's syndrome, evaporative dry eye, aqueous-deficient dry eye, biomarkers, Sjö test

\section{Introduction}

Sjögren's syndrome (SS) is a chronic and progressive systemic autoimmune disease that primarily involves immune-mediated damage to the lacrimal and salivary glands. ${ }^{1,2}$ This pathology translates into hallmark clinical symptoms of dry eyes (keratitis sicca or keratoconjunctivitis sicca) and dry mouth. SS is the second most prevalent autoimmune rheumatic disease, ${ }^{2}$ likely affecting close to four million Americans. ${ }^{3-5}$ The exact prevalence is difficult to determine due to variations in disease definitions and lack of a diagnostic gold standard. Women ${ }^{6}$ and Caucasians ${ }^{7}$ are the predominantly affected demographic groups. SS is primarily a disease of middle- to older aged adults, with onset typically occurring in the fourth or fifth decade of life. ${ }^{7}$ In one large Scandinavian study, the prevalence of SS was approximately seven times higher among elderly individuals (71-74 years of age) compared with a middle-aged population (40-44 years). ${ }^{8}$
Correspondence: Kenneth A Beckma Comprehensive EyeCare of Central Ohio, 450 Alkyre Run Drive \#I00, Westerville, $\mathrm{OH} 43082$, USA

Tel + I 6148905692

Fax +l 6148905629

Email kenbeckman22@aol.com 
The condition is associated with significant impairments in quality of life ${ }^{9-13}$ and functioning. ${ }^{11,14}$

While rheumatologists typically oversee the overall care of SS patients, a multidisciplinary team that involves ophthalmology specialists, as well as oral care professionals, is critical not just for the management of known SS patients, but also to identify potential SS cases. The symptoms of SS can progress slowly and are often highly variable in presentation, making diagnosis difficult. ${ }^{3,15-17}$ Consequently, it is estimated that the disease remains undiagnosed in more than half of affected adults. ${ }^{3,15,18}$ Data suggest that patients experience symptoms for an average of 3.9 years before a diagnosis of SS is made. ${ }^{5}$ Unfortunately, such delays in diagnosis can be a source of psychological distress from unexplained symptoms, ${ }^{12}$ not to mention prolonging the initiation of appropriate treatment. Early diagnosis and treatment of SS is essential to prevent or mitigate development of complications such as cerebrovascular events and myocardial infarction. ${ }^{12,15,16,19-22}$ Unfortunately, dry eye symptoms are very non-specific, especially in the immediate absence of other SS-related complaints or findings. In a recent US survey of eye care specialists, most practitioners reported relying primarily on patient history to guide treatment decisions. ${ }^{23}$ These findings underscore the need for reliable objective measures and diagnostic tools to quantify and classify dry eye symptoms.

This review aims to provide eye care professionals with an understanding of methods for distinguishing SS dry eye from other types of dry eye and to describe options for a differential diagnostic approach. The reader is reminded that SS is a systemic disease with many non-ocular manifestations that are not addressed in detail herein, but have been reviewed elsewhere. ${ }^{3}$ Collaboration with a rheumatologist is essential when SS is suspected in a given patient.

\section{Sjögren's syndrome}

SS can occur alone (primary SS) or in association with another underlying autoimmune disease (secondary SS), typically rheumatoid arthritis, systemic lupus erythematosus, systemic sclerosis (scleroderma), or polymyositis. ${ }^{7,15,24}$ The epidemiologic distribution of primary and secondary SS cases seems to be about even. ${ }^{5,25}$ Ocular and oral symptoms may be somewhat less severe in secondary SS. ${ }^{24}$ Primary SS presents the greatest diagnostic challenge, given that the disease presents de novo typically with gradual onset of vague, non-specific symptoms.

Primary SS is frequently associated with a wide range of systemic and extraglandular ocular complications, and these usually become evident an average of a decade after the onset of dry eye. ${ }^{21}$ A large retrospective cohort analysis found that primary SS was associated with an increased risk of cerebrovascular events and myocardial infarction. ${ }^{22}$ Patients with primary SS are more than twice as likely as age- and sex-matched controls to have hypertension and hypertriglyceridemia. High rates of depression have also been reported. ${ }^{12}$ Autonomic symptoms are common, affecting approximately half of patients with primary SS, and they are significantly correlated with overall symptom burden. ${ }^{26}$ In addition, approximately $20 \%-30 \%$ of patients with primary SS have clinical pulmonary involvement, which is associated with significantly greater impairment in quality of life/physical functioning, as well as with a fourfold increased 10-year mortality. ${ }^{27}$ Patients with primary SS have an estimated 16-37.5 times increased risk of lymphoma compared to the general population; ${ }^{28,29}$ the prevalence of non-Hodgkin B-cell lymphoma specifically is approximately $10 \%{ }^{7}$

\section{Dry eye disease}

Dry eye is one of two classic symptoms of SS, the other being dry mouth. While dry eye is a classic diagnostic feature of SS, it is a very common complaint with a wide range of underlying pathologies, thereby making it quite non-specific for SS by itself. ${ }^{9}$ The clinical consequences of dry eye include ocular discomfort, visual disturbance, tear film instability, and potential damage to the ocular surface. ${ }^{1}$ The symptoms of dry eye are usually accompanied by objective findings such as tear hyperosmolarity and ocular surface inflammation.

The differential diagnosis of ocular SS begins with a determination of the basic nature of the dry eye, namely aqueous-deficient dry eye (ADDE) or evaporative dry eye (EDE). ${ }^{1} \mathrm{SS}$ falls under the classification of ADDE; therefore, cases of dry eye initially classified as ADDE need to be further investigated as SS dry eye or non-SS dry eye (Figure 1). Steps for this process are described in the following sections, beginning with strategies for establishing and characterizing a broad diagnosis of dry eye, followed by more detailed methods of distinguishing the various sub-types of dry eye, and specific testing to help confirm a diagnosis of SS.

As described earlier, dry eye has been categorized based on pathology into two major classes: ADDE and EDE. ${ }^{1}$ ADDE refers primarily to a failure of lacrimal secretion, although a deficit in conjunctival water secretion may also contribute through decreased production of aqueous fluid by accessory lacrimal glands along the conjunctival surface. 


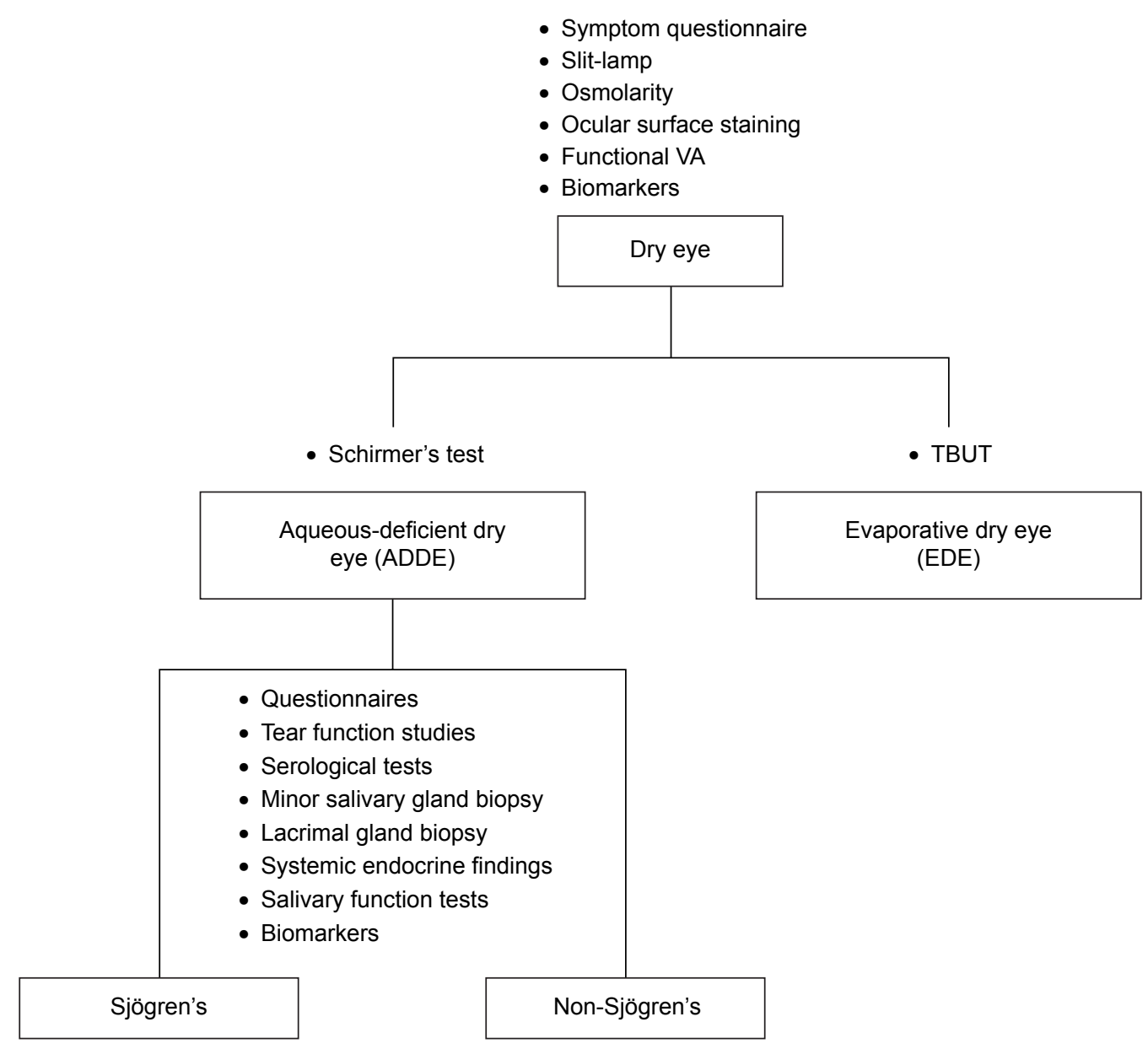

Figure I Overview of the differential diagnosis of dry eye secondary to Sjögren's syndrome.

Abbreviations: VA, visual acuity; TBUT, tear film break-up time.

ADDE, also called keratoconjunctivitis sicca (KCS), occurs in approximately $4 \%$ of adults over 65 years of age. ${ }^{30} \mathrm{EDE}$ is caused by excessive water loss from the ocular surface in patients with normal lacrimal secretion and may be a result of intrinsic (eg, meibomian oil deficiency) or extrinsic (eg, vitamin A deficiency, contact lens wear) factors. ${ }^{1}$

Despite these classifications and definitions, it is not uncommon for an individual patient to have dry eye caused by more than one mechanism, with subsequent triggering of additional dry eye pathology. Thus, many patients with dry eye have a combination of ADDE and EDE..$^{15,31}$

\section{General diagnosis of dry eye}

A number of tools can be used for the diagnosis of dry eye, including patient questionnaires, objective tests such as slitlamp evaluation, tear osmolarity assessments, ocular surface staining, functional visual acuity, tear meniscus evaluations, and inflammatory biomarker tests. Ideally, a combination of these assessments should be used.

\section{Symptom questionnaires}

The use of a structured symptom questionnaire is considered an excellent way to screen patients for dry eye disease, but should always be used in combination with objective clinical evaluation of dry eye status. ${ }^{31,32}$ There are several dry eye symptom questionnaires available with varying degrees of length and complexity. The following three-question, evidence-based symptom survey has been suggested as a simple clinical screening tool for dry eye disease; any "yes" response would trigger a more comprehensive dry eye work-up: ${ }^{31}$

1. How often do your eyes feel dryness, discomfort, or irritation? Would you say it is often or constantly? (Y/N)

2. When you have eye dryness, discomfort, or irritation, does this impact your activities (eg, do you stop or reduce your time doing them)? (Y/N)

3. Do you think you have dry eye? $(\mathrm{Y} / \mathrm{N})$

More extensive questionnaires are used primarily for clinical trials, including the Ocular Surface Disease Index 
$(\mathrm{OSDI})^{33}$ and the Standard Patient Evaluation of Eye Dryness questionnaire (SPEED). ${ }^{34}$

\section{Objective ocular testing}

Slit-lamp examination is recommended prior to any specific ocular testing; dry eye signs on slit-lamp examination include superficial corneal erosions, insufficient tear lake volume, early tear film break-up time (TBUT), conjunctival hyperemia, conjunctival surface irregularities, and meibomian gland dysfunction. ${ }^{35}$ Conjunctival scarring may also be seen.

Measurement of tear osmolarity was shown in a prospective, observational case series of 299 patients to have the highest positive predictive value $(86 \%)$ for the diagnosis of dry eye disease, higher than other objective tests. ${ }^{36} \mathrm{An}$ osmolarity threshold of $308 \mathrm{mOsms} / \mathrm{L}$ was noted as a sensitive cut-off for differentiating normal from mild to moderate dry eye disease. ${ }^{36}$ In a study comparing tear osmolarity in dry eye patients and normal controls, tear osmolarity of $305 \mathrm{mOsm} / \mathrm{L}$ was selected as the cut-off value for dry eye, $309 \mathrm{mOsm} / \mathrm{L}$ for moderate dry eye, $318 \mathrm{mOsm} / \mathrm{L}$ for severe dry eye (with area under the curve values of $0.737,0.759$, and 0.711 , respectively). ${ }^{37}$ Tear osmolarity testing is facilitated and simplified by the availability of commercial devices such as TearLab ${ }^{\circledR}$ (TearLab Corporation, San Diego, CA, USA). TearLab clinical usage guidelines suggest cut-off ranges of 300-320 mOsm/L for mild dry eye and 320-340 mOsm/L for moderate dry eye. ${ }^{38}$

Ocular surface staining patterns can help characterize dry eye and gauge severity. Staining can be performed using fluorescein, Rose Bengal, or lissamine green. Fluorescein concentrates in between living cells, thus stained areas represent an absence of epithelial cells, including cell death. While fluorescein is helpful in identifying superficial epithelial erosions, it is not a very sensitive or specific measure and cannot distinguish dry eye-related damage from other ocular injury. ${ }^{35,39}$ Rose Bengal and lissamine green stains concentrate in corneal and conjunctival cells that lack a healthy, protective mucin barrier, thereby identifying damaged epithelial cells (Figure 2). However, these tests also lack sensitivity or specificity for dry eye damage. Lissamine green is usually better tolerated than Rose Bengal. ${ }^{35}$

Other tests that may be performed include functional visual acuity and observation of the tear meniscus (tear lake). Functional visual acuity is a measure of visual acuity related to specific daily visual tasks, since patients with dry eye report decreased visual acuity during prolonged concentration on visual tasks such as reading, driving, or working at a computer. Unfortunately, testing of functional visual acuity is subjective and time-consuming.

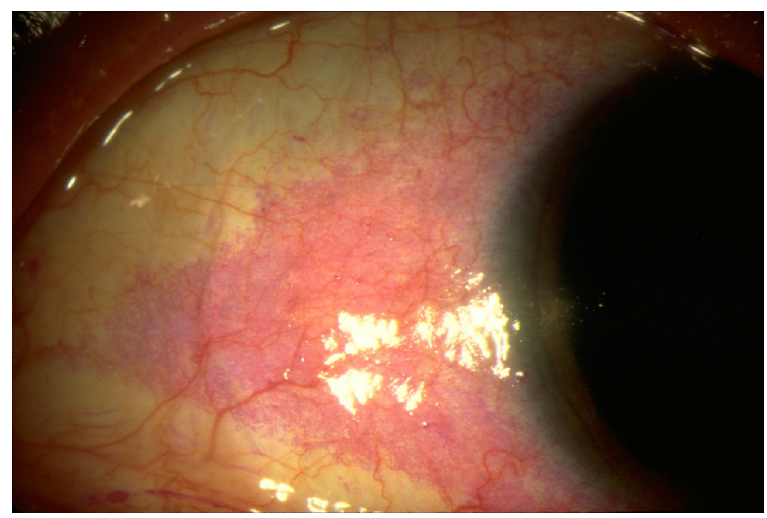

Figure 2 Ocular surface of a dry eye patient following Rose Bengal application. Notes: Purple stained areas indicate devitalized tissue and epithelial cell damage. Copyright American Academy of Ophthalmology 1994. Reproduced, with permission, from External Disease \& Cornea: A Multimedia Collection. Developed by Basic and Clinical Science Course Committee, Section 8, External Disease, American Academy of Ophthalmology, Copyright () 1994.

The tear meniscus reflects the amount of tears present where the bulbar conjunctiva and lower eyelid margin meet. When the height and curvature of the tear meniscus are measured, a patient with dry eye usually has a lower height than a patient without dry eye, though a low tear meniscus can occur in patients without dry eye. ${ }^{35}$

\section{Biomarkers and other emerging diagnostic techniques}

Patients with dry eye generally have higher levels of inflammatory markers than patients without dry eye. The inflammatory biomarker MMP-9 can be detected using the InflammaDry detector (Rapid Pathogen Screening, Inc., Sarasota, FL, USA), a point-of-care MMP-9 test, and it may facilitate making the diagnosis of dry eye. In one clinical trial, the InflammaDry Test was shown to have positive agreement of $86 \%$ and negative agreement of $97 \%$ with clinical assessment for the diagnosis of dry eye. ${ }^{40}$ Analysis of tear proteins such as lactoferrin can aid in the diagnosis of dry eye, since it has been shown that tear proteins are decreased in the tears of patients with ADDE, including those with SS and those without SS. ${ }^{41-43}$

There are a number of promising technologies in various stages of development for dry eye diagnosis, including reflective meniscometry, optical coherence tomography, interferometry, ocular surface thermography, and impression and brush cytology coupled with flow cytometry. ${ }^{32,35}$ Biopsies are seldom taken clinically.

\section{Differentiating between ADDE and EDE}

In patients who present with dry eye, it is important to differentiate between ADDE and EDE, because it is the 
presence of ADDE that raises the suspicion of SS. However, features of both types of dry eye are often present together. ${ }^{31}$

There are important differences in tear physiology between ADDE and EDE. There is significantly lower tear turnover in ADDE compared with EDE, while there is considerable overlap observed in tear evaporation between ADDE and EDE. However, there are no differences between the two major dry eye classifications with regard to tear osmolarity, volume, or distribution. ${ }^{44}$ Although the tear turnover rate has been reported as useful, it is cumbersome and not often used clinically. A cut-off value of $11 \% /$ minute for tear turnover rate had a sensitivity of $86 \%$ and specificity of $75 \%$; a cut-off of $60 \mathrm{~g} / \mathrm{m}^{2} \mathrm{~h}$ for tear evaporation had a sensitivity of $77 \%$ and a specificity of $58 \% .^{44}$

The Schirmer test, which measures total tear secretion, can be used to identify ADDE, the type of dry eye primarily associated with SS. In the absence of anesthesia, the Schirmer test measures reflex tearing, while the test performed with anesthesia measures basal tear secretion. ${ }^{31}$ A Schirmer test result of $<5 \mathrm{~mm}$ is indicative of aqueous deficiency, $>10 \mathrm{~mm}$ is normal, and $5-10 \mathrm{~mm}$ is considered borderline, but more suggestive of aqueous deficiency if done without anesthesia. ${ }^{32,35}$ The Schirmer II test is less commonly used; it involves stimulation of the nasal surface with a cotton tip applicator and subsequent measurement of reflex tear production. Drawbacks of the Schirmer test include the length of time required, patient discomfort, and the unreliable nature of the test. ${ }^{32,35}$

The TBUT is of some value in identifying tear film instability and aiding in the diagnosis of EDE. The established cutoff value for diagnosis of dry eye in general is $<10$ seconds, although lower cut-off values have been considered. ${ }^{32}$ Using this cut-off, the TBUT was shown to have a sensitivity of $82 \%$ and specificity of $86 \%$ for differentiating dry eye from non-dry eye. ${ }^{45}$ However, a more recent investigation found no difference in TBUT between patients with ADDE, EDE, and mixed (ADDE + EDE). ${ }^{46}$

\section{SS vs other types of ADDE}

Once ADDE has been diagnosed, it becomes important to differentiate between SS dry eye (primary vs secondary) and non-SS dry eye. There are many causes of non-SS ADDE, including primary or secondary lacrimal deficiency, lacrimal gland duct obstruction, reflex block (including post-LASIK), other neurotrophic corneas, and systemic drugs. Because SS typically presents in middle-aged women, symptoms such as cutaneous, oral, and vaginal dryness may be misattributed to menopause. ${ }^{3}$ Symptoms of dry eye and dry mouth may also be confused with atopy or anxiety. ${ }^{3}$ It is important to ask all patients who present with dry eye about concomitant dry mouth symptoms, as the combination is highly suggestive of SS and should prompt a full diagnostic evaluation. Combined symptoms of dry mouth, sore mouth, and dry eyes correctly classified $93 \%$ of patients with SS and $97.7 \%$ of controls in one study. ${ }^{47}$ However, it is possible for SS to present with only one of these findings, and the absence of dry mouth should not be the sole basis for ruling out additional work-up.

The many ocular symptoms of SS may include sensations of itching, grittiness, foreign body sensation, burning, and soreness despite a normal eye appearance. ${ }^{3,48}$ Patients may complain of photosensitivity, eye fatigue, erythema, reduced and fluctuating visual acuity, discharge, and the sensation of a film across the visual field. ${ }^{3,31}$ Some patients may not be able to tolerate contact lenses and may report needing to use tear substitutes. ${ }^{48}$ Accumulation of sticky mucus may make it difficult for patients to open their eyes in the morning. ${ }^{48}$ Ocular symptoms may be exacerbated by low humidity levels, exposure to cigarette smoke, anticholinergic drugs, antihistamines (as well as several other systemic medications), allergies, computer, tablet, and phone use, and topical drops and preservatives. ${ }^{3}$

Ocular findings upon examination of SS patients can include diminished tear secretion, although tear flow rates do not correlate with ocular discomfort, and accumulation of mucus secretions along the inner canthus. ${ }^{3,48}$ Desiccation may result in superficial/shallow erosions of the corneal epithelium; filamentary keratitis, revealed by slit-lamp examination, may occur in more severe cases. Some patients present with conjunctivitis due to infection with Staphylococcus aureus or other organisms. Rarely, enlargement of the lacrimal gland may be seen. Other potential ocular complications of SS include corneal ulceration, vascularization, opacification, and, in rare cases, perforation. . $^{3,48}$

\section{Full diagnostic work-up for SS}

Diagnostic criteria for SS were published in $2002^{49}$ (Table 1) and then updated in $2012^{50}$ (Table 2). The older criteria are more comprehensive, but more recent criteria are considered simpler to apply and rely on more objective measures, including biopsy. ${ }^{50}$ These criteria can help guide the diagnostic workup for SS, but may require the involvement of multiple specialists, including a rheumatologist. ${ }^{3}$

When SS is suspected in a patient with dry eye, an awareness of the extraocular signs and symptoms of SS can help make the diagnosis. ${ }^{48}$ These include oral manifestations such as inability to swallow dry food without liquid, 
Table I Revised international classification criteria for Sjögren's syndrome (American-European Consensus Group, 2002)

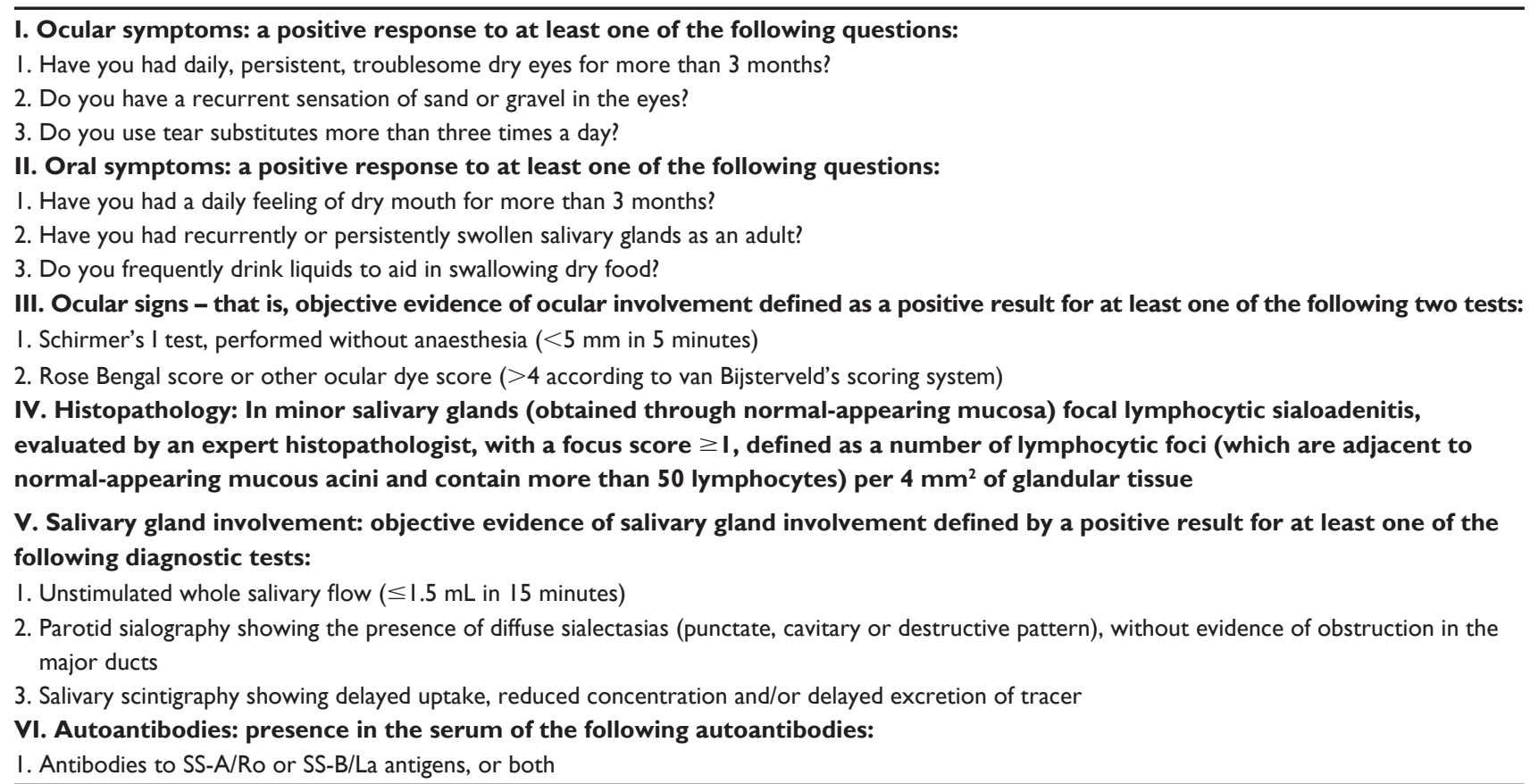

Note: Reproduced from Classification criteria for Sjögren's syndrome: a revised version of the European criteria proposed by the American-European Consensus Group, Vitali C, Bombardieri S, Jonsson R, et al, Ann Rheum Dis. 2002;61 (6):554-558,49 copyright $\odot 2002$ with permission from BMJ Publishing Group Ltd.

Abbreviations: SS-A/Ro, Sjögren's syndrome antigen type A; SS-B/La, Sjögren's syndrome antigen type B.

dry mouth, parotid swelling, dried and fissured tongue, and dental caries. Involvement of exocrine glands other than lacrimal and salivary glands can result in dry skin and hair, vaginal dryness, and gastrointestinal symptoms associated with impairment of protective mucus secretion. The systemic features of SS are varied and can include fatigue, arthritis (often misdiagnosed as rheumatoid arthritis), interstitial cystitis, neurological involvement, vasculopathies, interstitial pneumonitis, renal disease, lymphoma, and serological abnormalities.

The clinical work-up of SS typically involves a variety of tests, including tear function tests, serological tests for autoantibody biomarkers, minor salivary gland biopsy, lacrimal gland biopsy, systemic endocrine findings, and tests of salivary function (biscuit test, sialography). ${ }^{16,32,51-55}$ Questionnaires are of some value for the assessment of dry eye etiology, ${ }^{31,56}$ although questionnaires alone are insufficient to confirm a diagnosis of SS. The 5-item Dry Eye Questionnaire was validated in 2010; scores $>6$ indicate dry eye, and scores $>12$ may suggest further testing to rule out SS (Figure 3). ${ }^{57}$

Tear function tests have a role to play in the differential diagnosis of SS, with the most important aspect being differentiating between ADDE and EDE, as discussed earlier. The tear function index has been reported to be useful in the diagnosis of SS. ${ }^{58}$ In addition, Rose Bengal or lissamine green (vital dye stains) staining of the interpalpebral fissure is a noninvasive way to help diagnose $\mathrm{SS}$, because conjunctival staining may be seen earlier in the disease course. A normal TBUT rules out EDE, but a high percentage of patients with SS have a lid margin component (evaporative component). ${ }^{36}$ However, whether the TBUT is normal or rapid is not helpful for making the diagnosis of SS.

Table 2 Proposed classification criteria for Sjögren's syndrome (American College of Rheumatology, 20I2)

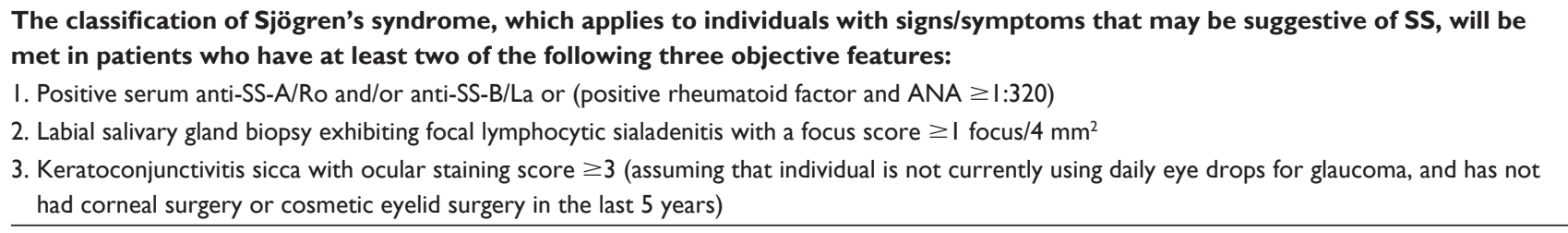

Note: Reproduced from Shiboski SC, Shiboski CH, Criswell L, et al. American College of Rheumatology classification criteria for Sjögren's syndrome: a data-driven, expert consensus approach in the Sjögren's International Collaborative Clinical Alliance cohort. Arthritis Care Res (Hoboken). 2012;64(4):475-487. ${ }^{50}$

Abbreviations: SS, Sjögren's syndrome; ANA, antinuclear antibody. 
1. Questions about EYE DISCOMFORT:

a. During a typical day in the past month, how often did your eyes feel discomfort?
0 Never
1 Rarely
2 Sometimes
3 Frequently
4 Constantly

b. When your eyes felt discomfort, how intense was this feeling of discomfort at the end of the day, within two hours of going to bed?

$\begin{array}{lccccc}\begin{array}{l}\text { Never } \\ \text { have it }\end{array} & \begin{array}{l}\text { Not at all } \\ \text { Intense }\end{array} & & & & \text { Very } \\ \mathbf{0} & \mathbf{1} & \mathbf{2} & \mathbf{3} & \mathbf{4} & \frac{\mathbf{5}}{\text { Intense }}\end{array}$

2. Questions about EYE DRYNESS:

a. During a typical day in the past month, how often did your eyes feel dry?
0 Never
1 Rarely
2 Sometimes
3 Frequently
4 Constantly

b. When your eyes felt dry, how intense was this feeling of dryness at the end of the day, within two hours of going to bed?

$\begin{array}{lccccc}\begin{array}{l}\text { Never } \\ \text { have it }\end{array} & \begin{array}{l}\text { Not at all } \\ \text { Intense }\end{array} & & & & \text { Very } \\ \mathbf{0} & \mathbf{1} & \mathbf{2} & \mathbf{3} & \mathbf{4} & \frac{\text { Intense }}{\mathbf{5}}\end{array}$

3. Question about WATERY EYES:

During a typical day in the past month, how often did your eyes look or feel excessively watery?

o Never

1 Rarely

2 Sometimes

3 Frequently

4 Constantly

Score: $1 \mathrm{a}+1 \mathrm{~b}+2 \mathrm{a}+2 \mathrm{~b}+3=$ Total

$++\ldots++$

Figure 3 The DEQ-5 (5-item Dry Eye Questionnaire), which is designed for patient self-assessment of dry eye severity on a typical day during the past month. Notes: A composite score $>6$ suggests dry eye. Copyright (C) Trustees of Indiana University, 2008, all rights reserved.

Evaluation of the salivary glands through biopsy or imaging is an important component of SS diagnosis. However, while biopsy of minor salivary glands is traditionally considered the gold standard for diagnosis of $\mathrm{SS},{ }^{3}$ it is not commonly performed in routine medical practice. Salivary gland ultrasonography has been shown to improve the diagnostic performance of the American College of Rheumatology classification criteria for SS (salivary gland ultrasonography $60.0 \%$ sensitive and $87.5 \%$ specific for SS $)^{59}$ and aid in the differential diagnosis of primary SS ${ }^{60}$ Acoustic radiation force impulse imaging of the parotid and submandibular glands may also help identify early-stage primary SS (diagnostic sensitivity, $81 \%$; specificity, $67 \%) .{ }^{61}$

\section{SS biomarkers}

Examination of biomarkers is a convenient and non-invasive way of evaluating patients for the presence of SS. Traditional biomarkers include SS-A/Ro, SS-B/La, ANA (antinuclear antibody), and RF (rheumatoid factor) ${ }^{50}$ (Table 3). It has been 
Table 3 Traditional and novel biomarkers for diagnosing Sjögren's syndrome

\section{Diagnostic characteristics}

Traditional biomarkers ${ }^{3}$

Anti-SS-A/Ro, anti-SS-B/La

Antinuclear antibody (ANA)

Rheumatoid factor (RF)

Novel biomarkers/autoantibodies ${ }^{62}$

Salivary protein-I (SP-I)

Carbonic anhydrase VI (CA-6)

Parotid secretory protein (PSP)
Not specific for SS; occurs in other autoimmune disorders, particularly SLE

Titer $\geq 1: 40$ present in approximately two-thirds of SS patients ${ }^{50}$

Found in many rheumatic conditions but is not unique to SS

Greatest sensitivity and specificity for early SS

Expressed very early in the course of SS; observed rarely in RA or normal controls Expressed early in SS; observed rarely in RA or normal controls

Abbreviations: RA, rheumatoid arthritis; SLE, systemic lupus erythematosus; SS, Sjögren's syndrome.

shown that patients expressing SS-A/Ro antibodies have an increased risk of developing extraglandular manifestations, such as cryoglobulinemia, anemia, vasculitis, leukopenia, and thrombocytopenia and thus warrant close monitoring. ${ }^{15}$ It appears that autoantibodies may be present long before symptom onset; a recent nested case control study from Sweden analyzed 175 serum samples drawn from 117 individuals prior to primary SS diagnosis; among cases which were autoantibody positive after SS diagnosis, at least one autoantibody specificity (ANA, RF, SS-A/Ro60, SS-A/Ro52, SS-B/La) was detected in $81 \%$ of cases at a median $4.3-5.1$ years preceding SS diagnosis, and as long as 20 years prior. ${ }^{63}$ In a smaller study, 29/44 (66\%) patients with primary SS had detectable autoantibodies as early as 18 years prior to symptom onset. ${ }^{64}$

Although SS-A/Ro, SS-B/La, ANA, and RF are considered important in the diagnosis of SS, they are not always positive in patients with SS, especially in early cases. In a large, prospective cohort of patients at optometry and ophthalmology centers within the USA presenting with clinically significant ADDE, 38/327 (11.6\%) patients were found to have SS. ${ }^{25}$ Compared with patients without SS, those with SS demonstrated significantly worse conjunctival and corneal staining, Schirmer's test results, and symptoms. Primary SS was significantly more likely in patients positive for ANA or RF. However, SS-A/Ro and SS-B/La are positive in only approximately half of patients with SS who present to ophthalmology clinics primarily with dry eye symptoms. ${ }^{15}$ The authors suggest that patients with clinically significant dry eye signs and symptoms plus ANA and/or RF positivity should be offered diagnostic testing for SS even in the absence of positive SS-A/SS-B results. ${ }^{25}$

Antibody detection rates may vary by the type of diagnostic test used (ELISA, double immunodiffusion, Western blot, and addressable laser bead assay). For example, in one study, immunodot was able to detect anti-Ro52/60 antibodies in approximately $20 \%$ of suspected SS cases who tested negative for anti-SS-A/SS-B findings using ELISA; however, immunodot is expensive and time-consuming. ${ }^{65}$
Using more than one detection method may improve diagnostic success.

Based on findings in mouse models, it has been hypothesized that SS begins as an organ-specific disease that initially affects the lacrimal and submandibular glands and then progresses to involve the other salivary glands; it has been further postulated that identification of organ-specific antibodies in the lacrimal and major salivary glands may lead to early diagnosis of SS. ${ }^{66}$ Recent work has identified autoantibodies to salivary gland protein-1 (SP1), parotid secretory protein (PSP), and carbonic anhydrase VI (CA6) in patients with a $>5$-year history of SS. ${ }^{62,67}$ Overall, $54 \%$ of the SS patients had autoantibodies to SP1, $54 \%$ to CA6, and $18 \%$ to PSP; $69 \%$ had autoantibodies to either SP1 or CA6, while $62 \%$ had antibodies to Ro or La, the traditional markers. Upon examination of patients who met the criteria for SS but who lacked antibodies to Ro or La, $45 \%$ had autoantibodies to the three novel biomarkers. In addition, among patients with symptoms suggestive of SS for less than 2 years, only $31 \%$ were positive for antibodies to Ro or La, while $76 \%$ were positive for antibodies to CA6 or SP $1 .{ }^{62}$ In another study, patients with different severities of SS were evaluated, and the novel biomarkers were found to be associated with early disease, with many patients who lacked antibodies to the traditional markers being positive for anti-SP1, anti-CA6, and anti-PSP. ${ }^{67}$ Thus, these novel biomarkers can be considered as useful markers of early SS.

The $\mathrm{Sjö}^{\circledR}$ test (Bausch \& Lomb, Rochester, NY, USA) is an advanced, commercially available panel for the early detection of SS, requiring a blood sample obtained by an in-office finger prick or blood collection at a diagnostic laboratory. It includes the early biomarkers SP1, PSP, and CA6, along with the classic Sjögren's biomarkers SS-A/Ro, SS-B/La, ANA, and RF. This combination of biomarkers provides greater sensitivity and specificity compared to traditional testing. The cumulative sensitivity of the Sjö panel was $89.9 \%$ as determined from four clinical studies with a total of 248 sera samples. Based on sera samples from 79 age- and sex-matched controls and 64 pediatric controls, the cumulative specificities 
of the entire $\mathrm{Sjö} \mathrm{panel} \mathrm{and} \mathrm{the} \mathrm{novel} \mathrm{early} \mathrm{biomarkers} \mathrm{were}$ $78.7 \%$ and $82.5 \%$, respectively (personal communication, Dr Lakshmanan Suresh, Immco Diagnostics, Buffalo, NY, USA). Further, incorporation of the early biomarkers into the $\mathrm{Sjö}$ test facilitates the diagnosis of SS in patients with chronic dry eye who previously tested negative for traditional anti-Ro/SS-A and anti-La/SS-B biomarkers. ${ }^{67-70}$ The advent of advanced and easy to use tests such as this may improve diagnostic accuracy in suspected cases of SS and allow for disease identification at earlier stages.

Several additional novel biomarkers of SS have been identified, ${ }^{71,72}$ but are not yet being used routinely in clinical practice. Anti-kallikrein (KLK) antibodies were found to be significantly higher in the sera of SS patients compared with non-SS dry eye patients or normal controls; using an optical density cut-off of 0.2695 , anti-KLK11 antibody distinguished the SS group from non-SS dry eye and controls with a sensitivity of $82 \%$ and a specificity of $94 \% .{ }^{73}$ Tear cathepsin $\mathrm{S}$ activity is similar in patients with primary and secondary SS but is much higher in SS than in patients with non-specific dry eye disorder or normal controls; very high tear cathepsin S activity may be considered suggestive of SS. ${ }^{74}$ IgG and IgA antibodies against a-fodrin may be considered activation markers of SS. ${ }^{75}$ Lymphotoxin $\alpha$ has also been shown to be elevated in salivary gland secretions and sera of patients with $\mathrm{SS}^{76}$

\section{Conclusion}

Patient complaints and clinical findings suggestive of dry eye, especially ADDE, should always trigger a suspicion of $\mathrm{SS}$ and prompt further investigation, including queries about concomitant dry mouth symptoms. All patients with clinically significant ADDE should be considered for serological assessment for SS, given the availability of new serological diagnostic tests and the potentially serious consequences of missing the diagnosis. Vigilance and proactive steps on the part of eye care professionals can play a critical role in facilitating the early recognition of SS and referral to a rheumatologist, enabling timely intervention for both ocular and non-ocular manifestations.

\section{Acknowledgments}

Editorial assistance for this paper was provided by the staff of Churchill Communications (Maplewood, NJ, USA) with funding from Bausch \& Lomb.

\section{Disclosure}

KA Beckman is a paid consultant for Allergan, Bausch \& Lomb, Shire, and TearLab; a paid speaker for Allergan and
Shire; and an investor in RPS. J Luchs is a paid consultant for Allergan, Alcon, Bausch \& Lomb, Shire, Doctor's Allergy, Tear Lab, Auven, Omeros, AMO; serves on a Speaker's Bureau for Allergan, Bausch \& Lomb, Shire, Doctor's Allergy, Tear Lab; conducts research for Allergan, Alcon, Bausch \& Lomb, Shire, Eleven, Aerie, Auven, Ocular Therapeutics, Topokine, Inotek, Kala, Refocus; and has equity in Insightful Solutions, CXLO, RPS, Calhoun Vision, and Omega Ophthalmics. MS Milner has served as a paid speaker for Allergan, Shire, Bausch \& Lomb, and TearScience; has served as a paid consultant/advisory board member for Allergan and Shire; and is an investor in RPS. The authors report no other conflicts of interest in this work.

\section{References}

1. Anonymous. The definition and classification of dry eye disease: report of the Definition and Classification Subcommittee of the International Dry Eye WorkShop (2007). Ocul Surf. 2007;5(2):75-92.

2. Tincani A, Andreoli L, Cavazzana I, et al. Novel aspects of Sjögren's syndrome in 2012. BMC Med. 2013;11:93.

3. Kassan SS, Moutsopoulos HM. Clinical manifestations and early diagnosis of Sjögren syndrome. Arch Intern Med. 2004;164(12): $1275-1284$.

4. Helmick CG, Felson DT, Lawrence RC, et al. Estimates of the prevalence of arthritis and other rheumatic conditions in the United States. Part I. Arthritis Rheum. 2008;58(1):15-25.

5. Sjögren's Syndrome Foundation. About Sjögren's syndrome. Available from: https://www.sjogrens.org/home/about-sjogrens-syndrome. Accessed May 26, 2015.

6. Pillemer SR, Matteson EL, Jacobsson LT, et al. Incidence of physiciandiagnosed primary Sjögren syndrome in residents of Olmsted County, Minnesota. Mayo Clin Proc. 2001;76(6):593-599.

7. Patel R, Shahane A. The epidemiology of Sjögren's syndrome. Clin Epidemiol. 2014;6:247-255.

8. Haugen AJ, Peen E, Hultén B, et al. Estimation of the prevalence of primary Sjögren's syndrome in two age-different community-based populations using two sets of classification criteria: the Hordaland Health Study. Scand J Rheumatol. 2008;37(1):30-34.

9. No authors listed. The epidemiology of dry eye disease: report of the Epidemiology Subcommittee of the International Dry EyeWorkShop (2007). Ocul Surf. 2007;5(2):93-107.

10. López-Jornet P, Camacho-Alonso F. Quality of life in patients with Sjögren's syndrome and sicca complex. J Oral Rehabil. 2008;35(12): 875-881.

11. Meijer JM, Meiners PM, Huddleston Slater JJ, et al. Health-related quality of life, employment and disability in patients with Sjögren's syndrome. Rheumatology (Oxford). 2009;48(9):1077-1082.

12. Segal B, Bowman SJ, Fox PC, et al. Primary Sjögren's Syndrome: health experiences and predictors of health quality among patients in the United States. Health Qual Life Outcomes. 2009;7:46.

13. Lendrem D, Mitchell S, McMeekin P, et al. Health-related utility values of patients with primary Sjögren's syndrome and its predictors. Ann Rheum Dis. 2014;73(7):1362-1368.

14. Hackett KL, Newton JL, Frith J, et al. Impaired functional status in primary Sjögren's syndrome. Arthritis Care Res (Hoboken). 2012;64(11): $1760-1764$.

15. Akpek EK, Klimava A, Thorne JE, Martin D, Lekhanont K, Ostrovsky A. Evaluation of patients with dry eye for presence of underlying Sjögren syndrome. Cornea. 2009;28(5):493-497.

16. Gomes Pde S, Juodzbalys G, Fernandes MH, Guobis Z. Diagnostic approaches to Sjögren's syndrome: a literature review and own clinical experience. J Oral Maxillofac Res. 2012;3(1):e3. 
17. Hernández-Molina G, Sánchez-Hernández T. Clinimetric methods in Sjögren's syndrome. Semin Arthritis Rheum. 2013;42(6):627-639.

18. Usuba FS, Lopes JB, Fuller R, et al. Sjögren's syndrome: an underdiagnosed condition in mixed connective tissue disease. Clinics (Sao Paulo). 2014;69(3):158-162.

19. Meiners P, Vissink A, Kroese F, et al. ABATACEPT treatment reduces disease activity in early primary Sjögren's syndrome (Phase II Open Label ASAP Study). Ann Rheum Dis. 2014;73(7):1393-1396.

20. Juarez M, Toms TE, de Pablo P, et al. Cardiovascular risk factors in women with primary Sjögren's syndrome: United Kingdom primary Sjögren's syndrome registry results. Arthritis Care Res (Hoboken). 2014; 66(5):757-764.

21. Akpek EK, Mathews $\mathrm{P}, \mathrm{Hahn} \mathrm{S}$, et al. Ocular and systemic morbidity in a longitudinal cohort of Sjögren's syndrome. Ophthalmology. 2015; 122(1):56-61.

22. Bartoloni E, Baldini C, Schillaci G, et al. Cardiovascular disease risk burden in primary Sjögren's syndrome: results of a population-based multicentre cohort study. J Intern Med. 2015;278(2):185-192.

23. Williamson JF, Huynh K, Weaver MA, Davis RM. Perceptions of dry eye disease management in current clinical practice. Eye Contact Lens. 2014;40(2):111-115.

24. National Institute of Arthritis and Musculoskeletal and Skin Diseases. Sjogren's Syndrome. June 2103. Available from: http://www.niams. nih.gov/health_info/Sjogrens_Syndrome/default.asp\#versus. Accessed August 5, 2015.

25. Liew MS, Zhang M, Kim E, Akpek EK. Prevalence and predictors of Sjögren's syndrome in a prospective cohort of patients with aqueousdeficient dry eye. Br J Ophthalmol. 2012;96(12):1498-1503.

26. Newton JL, Frith J, Powell D, et al. Autonomic symptoms are common and are associated with overall symptom burden and disease activity in primary Sjögren's syndrome. Ann Rheum Dis. 2012;71(12): 1973-1979.

27. Palm O, Garen T, Berge Enger T, et al. Clinical pulmonary involvement in primary Sjögren's syndrome: prevalence, quality of life and mortality a retrospective study based on registry data. Rheumatology (Oxford). 2013;52(1):173-179.

28. Solans-Laque R, Lopez-Hernandez A, Bosch-Gil J, Palacios A, Campillo M, Vilardell-Tarres M. Risk, predictors, and clinical characteristics of lymphoma development in primary Sjögren's syndrome. Semin Arthritis Rheum. 2011;41(3):415-423.

29. Lazarus MN, Robinson D, Mak V, Moller H, Isenberg DA. Incidence of cancer in a cohort of patients with primary Sjögren's syndrome. Rheumatology (Oxford). 2006;45(8):1012-1015.

30. Liu KC, Huynh K, Grubbs J Jr, Davis RM. Autoimmunity in the pathogenesis and treatment of keratoconjunctivitis sicca. Curr Allergy Asthma Rep. 2014;14(1):403.

31. Foulks GN, Forstot SL, Donshik PC, et al. Clinical guidelines for management of dry eye associated with Sjögren disease. Ocul Surf. $2015 ; 13(2): 118-132$.

32. No authors listed. Methodologies to diagnose and monitor dry eye disease: report of the Diagnostic Methodology Subcommittee of the International Dry Eye WorkShop (2007). Ocul Surf. 2007;5(2):108-152.

33. Walt J, Rowe M, Stern K. Evaluating the functional impact of dry eye: the Ocular Surface Disease Index (Abstract). Drug Inf J. 1997;31: 1436.

34. Ngo W, Situ P, Keir N, Korb D, Blackie C, Simpson T. Psychometric properties and validation of the Standard Patient Evaluation of Eye Dryness questionnaire. Cornea. 2013;32(9):1204-1210.

35. Zeev MS, Miller DD, Latkany R. Diagnosis of dry eye disease and emerging technologies. Clin Ophthalmol. 2014;8:581-590.

36. Lemp MA, Bron AJ, Baudouin C, et al. Tear osmolarity in the diagnosis and management of dry eye disease. Am J Ophthalmol. 2011;151(5): 792-798.e1. doi:10.1016/j.ajo.2010.10.032. Epub 2011 Feb 18.

37. Versura P, Profazio V, Campos EC. Performance of tear osmolarity compared to previous diagnostic tests for dry eye diseases. Curr Eye Res. 2010;35(7):553-564.
38. Clinical Workflow. TearLab Inc. Available from: http://www.tearlab. com/products/doctors/practice.htm. Accessed August 12, 2015.

39. Abelson MB, Ingerman A. The dye-namics of dry-eye diagnosis. Rev Ophthalmol. November 15, 2005. Available from: http://www. reviewofophthalmology.com/content/d/therapeutic_topics/i/1311/ c/25254/. Accessed August 5, 2015.

40. Sambursky R, Davitt WF III, Friedberg M, Tauber S. Prospective, multicenter, clinical evaluation of point-of-care matrix metalloproteinase-9 test for confirming dry eye disease. Cornea. 2014;33(8):812-818.

41. Ohashi Y, Ishida R, Kojima T, et al. Abnormal protein profiles in tears with dry eye syndrome. Am J Ophthalmol. 2003;136(2):291-299.

42. Versura P, Bavelloni A, Grillini M, Fresina M, Campos EC. Diagnostic performance of a tear protein panel in early dry eye. Mol Vis. 2013;19: $1247-1257$.

43. Karns K, Herr AE. Human tear protein analysis enabled by an alkaline microfluidic homogeneous immunoassay. Anal Chem. 2011;83(21): $8115-8122$.

44. Khanal S, Tomlinson A, Diaper CJ. Tear physiology of aqueous deficiency and evaporative dry eye. Optom Vis Sci. 2009;86(11): $1235-1240$.

45. Mengher LS, Pandher KS, Bron AJ. Non-invasive tear film break up time; sensitivity and specificity. Acta Ophthalmol. 1986;64:441-444.

46. Lemp MA, Crews LA, Bron AJ, Foulks GN, Sullivan BD. Distribution of aqueous-deficient and evaporative dry eye in a clinic-based patient cohort: a retrospective study. Cornea. 2012;31(5):472-478.

47. Al-Hashimi I, Khuder S, Haghighat N, Zipp M. Frequency and predictive value of the clinical manifestations in Sjögren's syndrome. J Oral Pathol Med. 2001;30:1-16.

48. Venables PJ. Sjögren's syndrome. Best Pract Res Clin Rheumatol. 2004;18(3):313-329.

49. Vitali C, Bombardieri S, Jonsson R, et al. Classification criteria for Sjögren's syndrome: a revised version of the European criteria proposed by the American-European Consensus Group. Ann Rheum Dis. 2002; 61(6):554-558.

50. Shiboski SC, Shiboski CH, Criswell L, et al. American College of Rheumatology classification criteria for Sjögren's syndrome: a datadriven, expert consensus approach in the Sjögren's International Collaborative Clinical Alliance cohort. Arthritis Care Res (Hoboken). 2012; 64(4):475-487.

51. Versura P, Frigato M, Cellini M, Mulè R, Malavolta N, Campos EC. Diagnostic performance of tear function tests in Sjögren's syndrome patients. Eye (Lond). 2007;21(2):229-237.

52. Villani E, Galimberti D, Viola F, Mapelli C, Ratiglia R. The cornea in Sjögren's syndrome: an in vivo confocal study. Invest Ophthalmol Vis Sci. 2007;48(5):2017-2022.

53. Kojima T, Matsumoto Y, Dogru M, Tsubota K. The application of in vivo laser scanning confocal microscopy as a tool of conjunctival in vivo cytology in the diagnosis of dry eye ocular surface disease. Mol Vis. 2010; 16:2457-2464.

54. Wakamatsu TH, Sato EA, Matsumoto Y, et al. Conjunctival in vivo confocal scanning laser microscopy in patients with Sjögren syndrome. Invest Ophthalmol Vis Sci. 2010;51(1):144-150.

55. Machetta F, Fea AM, Actis AG, de Sanctis U, Dalmasso P, Grignolo FM. In vivo confocal microscopic evaluation of corneal langerhans cells in dry eye patients. Open Ophthalmol J. 2014;8:51-59.

56. Amparo F, Schaumberg DA, Dana R. Comparison of two questionnaires for dry eye symptom assessment: the ocular surface disease index and the symptom assessment in dry eye. Ophthalmology. 2015;122(7): $1498-1503$.

57. Chalmers RL, Begley CG, Caffery B. Validation of the 5-Item Dry Eye Questionnaire (DEQ-5): discrimination across self-assessed severity and aqueous tear deficient dry eye diagnoses. Cont Lens Anterior Eye. 2010;33(2):55-60.

58. Kaye SB, Sims G, Willoughby C, Field AE, Longman L, Brown MC. Modification of the tear function index and its use in the diagnosis of Sjögren's syndrome. Br J Ophthalmol. 2001;85(2):193-199. 
59. Cornec D, Jousse-Joulin S, Marhadour T, et al. Salivary gland ultrasonography improves the diagnostic performance of the 2012 American College of Rheumatology classification criteria for Sjögren's syndrome. Rheumatology (Oxford). 2014;53(9):1604-1607.

60. Hammenfors DS, Brun JG, Jonsson R, Jonsson MV. Diagnostic utility of major salivary gland ultrasonography in primary Sjögren's syndrome. Clin Exp Rheumatol. 2015;33(1):56-62.

61. Knopf A, Hofauer B, Thürmel K, et al. Diagnostic utility of Acoustic Radiation Force Impulse (ARFI) imaging in primary Sjoegren's syndrome. Eur Radiol. 2015 Apr 11 [Epub ahead of print].

62. Shen L, Suresh L, Lindemann M, et al. Novel autoantibodies in Sjögren's syndrome. Clin Immunol. 2012;145(3):251-255.

63. Theander E, Jonsson R, Sjostrom B, Brokstad K, Olsson P, Henriksson G. Prediction of Sjogren's syndrome years before diagnosis and identification of patients with early onset and severe disease course by autoantibody profiling. Arthritis Rheumatol. 2015;67(9):2427-2436.

64. Jonsson R, Theander E, Sjöström B, Brokstad K, Henriksson G. Autoantibodies present before symptom onset in primary Sjögren syndrome. JAMA. 2013;310(17):1854-1855.

65. Mekinian A, Nicaise-Roland P, Chollet-Martin S, Fain O, Crestani B. Anti-SSA Ro52/Ro60 antibody testing by immunodot could help the diagnosis of Sjögren's syndrome in the absence of anti-SSA/ SSB antibodies by ELISA. Rheumatology (Oxford). 2013;52(12): 2223-2228.

66. Xuan J, Shen L, Malyavantham K, Pankewycz O, Ambrus JL Jr, Suresh L. Temporal histological changes in lacrimal and major salivary glands in mouse models of Sjögren's syndrome. BMC Oral Health. 2013;13:51.

67. Suresh L, Malyavantham K, Shen L, Ambrus JL Jr. Investigation of novel autoantibodies in Sjögren's syndrome utilizing Sera from the Sjögren's international collaborative clinical alliance cohort. BMC Ophthalmol. 2015;15(1):38.
68. Beckman KA. Detection of early markers for Sjögren syndrome in dry eye patients. Cornea. 2014;33(12):1262-1264.

69. Vishwanath S, Everett S, Shen L, Malyavantham K, Suresh L, Ambrus JL Jr. Xerophthalmia of Sjögren's Syndrome Diagnosed with Anti-Salivary Gland Protein 1 Antibodies. Case Rep Ophthalmol. 2014; 5(2):186-189.

70. Vishwanath S, Shen L, Suresh L, Ambrus JL Jr. Anti-salivary gland protein 1 antibodies in two patients with Sjögren's syndrome: two case reports. J Med Case Rep. 2014;8:145.

71. Giusti L, Baldini C, Bazzichi L, Bombardieri S, Lucacchini A. Proteomic diagnosis of Sjögren's syndrome. Expert Rev Proteomics. 2007;4(6): 757-767.

72. Zhou L, Wei R, Zhao P, Koh SK, Beuerman RW, Ding C. Proteomic analysis revealed the altered tear protein profile in a rabbit model of Sjögren's syndrome-associated dry eye. Proteomics. 2013;13(16): 2469-2481.

73. El Annan J, Jiang G, Wang D, Zhou J, Foulks GN, Shao H. Elevated immunoglobulin to tissue KLK11 in patients with Sjögren syndrome. Cornea. 2013;32(5):e90-e93.

74. Hamm-Alvarez SF, Janga SR, Edman MC, et al. Tear cathepsin S as a candidate biomarker for Sjögren's syndrome. Arthritis Rheumatol. 2014 66(7):1872-8181

75. Yavuz S, Toker E, Bicakcigil M, Mumcu G, Cakir S. Comparative analysis of autoantibodies against a-fodrin in serum, tear fluid, and saliva from patients with Sjögren's syndrome. J Rheumatol. 2006;33(7): 1289-1292.

76. Shen L, Suresh L, Wu J, et al. A role for lymphotoxin in primary Sjögren's disease. J Immunol. 2010;185(10):6355-6363.
Clinical Ophthalmology

\section{Publish your work in this journal}

Clinical Ophthalmology is an international, peer-reviewed journa covering all subspecialties within ophthalmology. Key topics include: Optometry; Visual science; Pharmacology and drug therapy in eye diseases; Basic Sciences; Primary and Secondary eye care; Patien Safety and Quality of Care Improvements. This journal is indexed on

Submit your manuscript here: http://www.dovepress.com/clinical-ophthalmology-journal

\section{Dovepress}

PubMed Central and CAS, and is the official journal of The Society of Clinical Ophthalmology (SCO). The manuscript management system is completely online and includes a very quick and fair peer-review system, which is all easy to use. Visit http://www.dovepress.com/ testimonials.php to read real quotes from published authors. 